\title{
Neoadjuvant chemotherapy with S-1 and surgical resection for a mucinous gastric cancer with peritoneal dissemination
}

\author{
Madoka Hamada $^{1}$, Akihito Tsuji ${ }^{2}$, Jun Imata ${ }^{3}$, Yutaka Nishioka ${ }^{1}$, Kazuhide Ozaki $^{1}$, Yasuo Shima ${ }^{1}$, \\ and TADASHI HORIMI ${ }^{1}$ \\ ${ }^{1}$ Department of Surgery, Kochi Municipal Central Hospital, 2-7-33 Sakurai-cho, Kochi 780-0821, Japan \\ ${ }^{2}$ Department of Medicine, Kochi Municipal Central Hospital, Kochi, Japan \\ ${ }^{3}$ Department of Pathology, Kochi Municipal Central Hospital, Kochi, Japan
}

\begin{abstract}
We herein report the case of a patient with mucinous gastric carcinoma with peritoneal dissemination that disappeared after neoadjuvant chemotherapy with $S-1$ alone. The patient has survived for over 23 months after surgery, without recurrence. A 60-year old man was referred to our hospital because of an advanced gastric cancer, detected by upper gastrointestinal endoscopy at another hospital. Staging laparoscopy was performed on October 25, 2002, and revealed massive peritoneal dissemination. Two courses of neoadjuvant chemotherapy with S-1 were administered, at $120 \mathrm{mg} /$ day for 28 days, as one course. Total gastrectomy, with $\mathrm{D2}$ lymph node dissection, was performed on January 24, 2003. The peritoneal dissemination had macroscopically disappeared and the cytology of the peritoneal lavage fluid was class III. His final diagnosis was gastric carcinoma, MLU, type 3, T2(SS), P0, H0, M0, N3, CY0, stage IV.
\end{abstract}

Key words Gastric cancer · Peritoneal dissemination · S-1 · Neoadjuvant chemotherapy

\section{Introduction}

The 5-year survival of patients with stage IV gastric cancer in Japan has been reported to be $16.6 \%$ [1]. Improvements in therapeutic strategies have been strongly suggested. Most advanced gastric carcinomas progress, or they recur with peritoneal dissemination. Median survival time for patients with peritoneal dissemination after surgery is reported to be 10 months [2,3]. S-1 (Taiho Pharmaceutical, Tokyo, Japan) is a new anticancer drug that has been reported to have a response rate as high as $40 \%-50 \%$ in advanced gastric carcinomas. Furthermore, the response rate has been reported to be as high as $70 \%$ in combination with

Offprint requests to: $\mathrm{M}$. Hamada

Received: May 18, 2004 / Accepted: October 25, 2004 cisplatin (CDDP). Excellent clinical and experimental results have been reported with S-1 for peritoneal dissemination [4], but long-term survival without recurrence is rare, as only a few cases of long-term survival have been reported. Curative surgery is the recommended treatment for gastric cancer. The results of surgery without curability for stage IV patients are generally dismal. It is not known whether or not adjuvant chemotherapy with new anticancer agents such as S-1 is beneficial for the survival of patients with peritoneal dissemination. As curative surgery is considered to be the best therapy for gastric cancer, downstaging with adjuvant chemotherapy and curative surgery is a possibility to improve the prognosis of stage IV gastric cancer patients, after precise preoperative staging.

We report the case of a patient with a gastric carcinoma in which peritoneal dissemination disappeared after neoadjuvant chemotherapy with S-1 alone. The patient has survived for over 23 months since the operation, without evidence of recurrence.

\section{Case report}

A 60-year-old man with anorexia was referred to our hospital, with advanced gastric cancer, detected at an other hospital by upper gastrointestinal endoscopy. Preoperative abdominal computed tomography (CT) scan showed a small amount of ascites and an infiltrative soft-tissue lesion in the peritoneal fat or on the peritoneal surface, suggesting peritoneal dissemination (Fig. 1A). CT also showed lymphadenopathy, which was indicative of metastases, on the lesser curvature of the stomach. Physical examination revealed no specific problems. The serum carcinoembryonic antigen (CEA) level was $1.2 \mathrm{ng} / \mathrm{ml}$ and the carbohydrate antigen (CA) 19-9 level was $5.7 \mathrm{U} / \mathrm{ml}$.

Gastrointestinal endoscopy revealed that the tumor was located on the greater curvature of the stomach, 

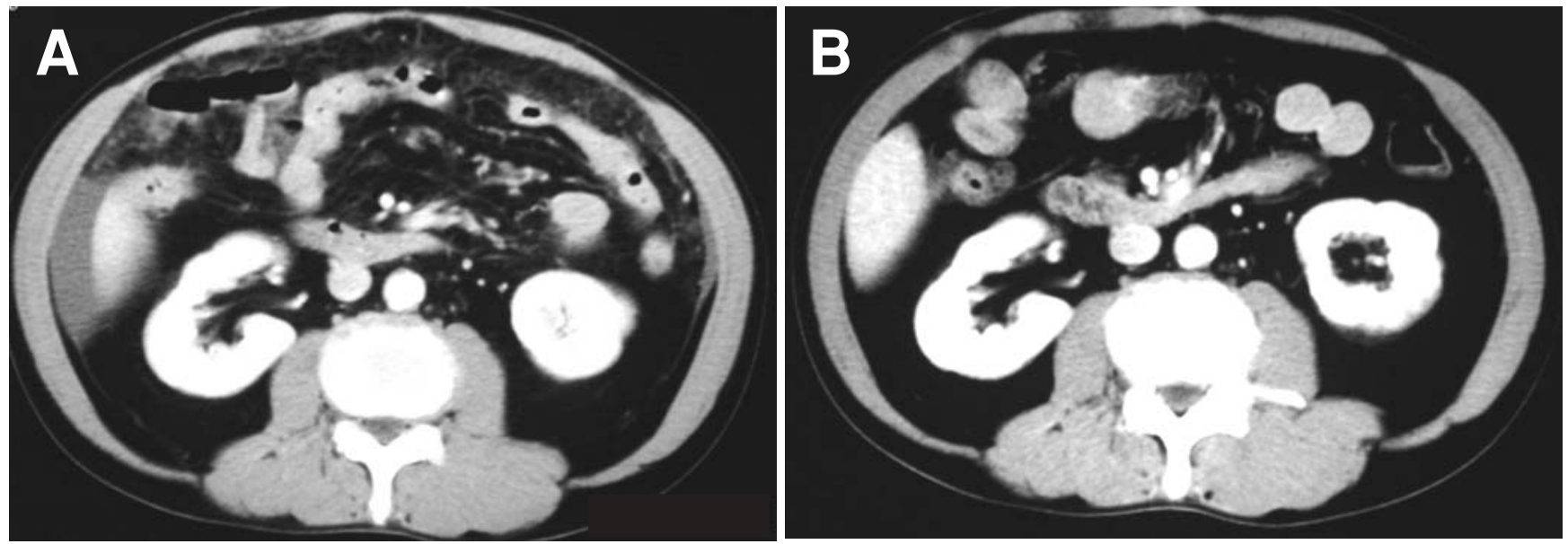

Fig. 1. A Abdominal computed tomography (CT) scan before chemotherapy. A small amount of ascites is collected around the liver, and there is an infiltrative soft-tissue lesion in the peritoneal fat or on the peritoneal surface, suggesting peritoneal dissemination around the mesentery or omentum. B Abdominal CT scan after chemotherapy. Fluid collection is not observed in the abdominal cavity. Peritoneal dissemination is markedly diminished
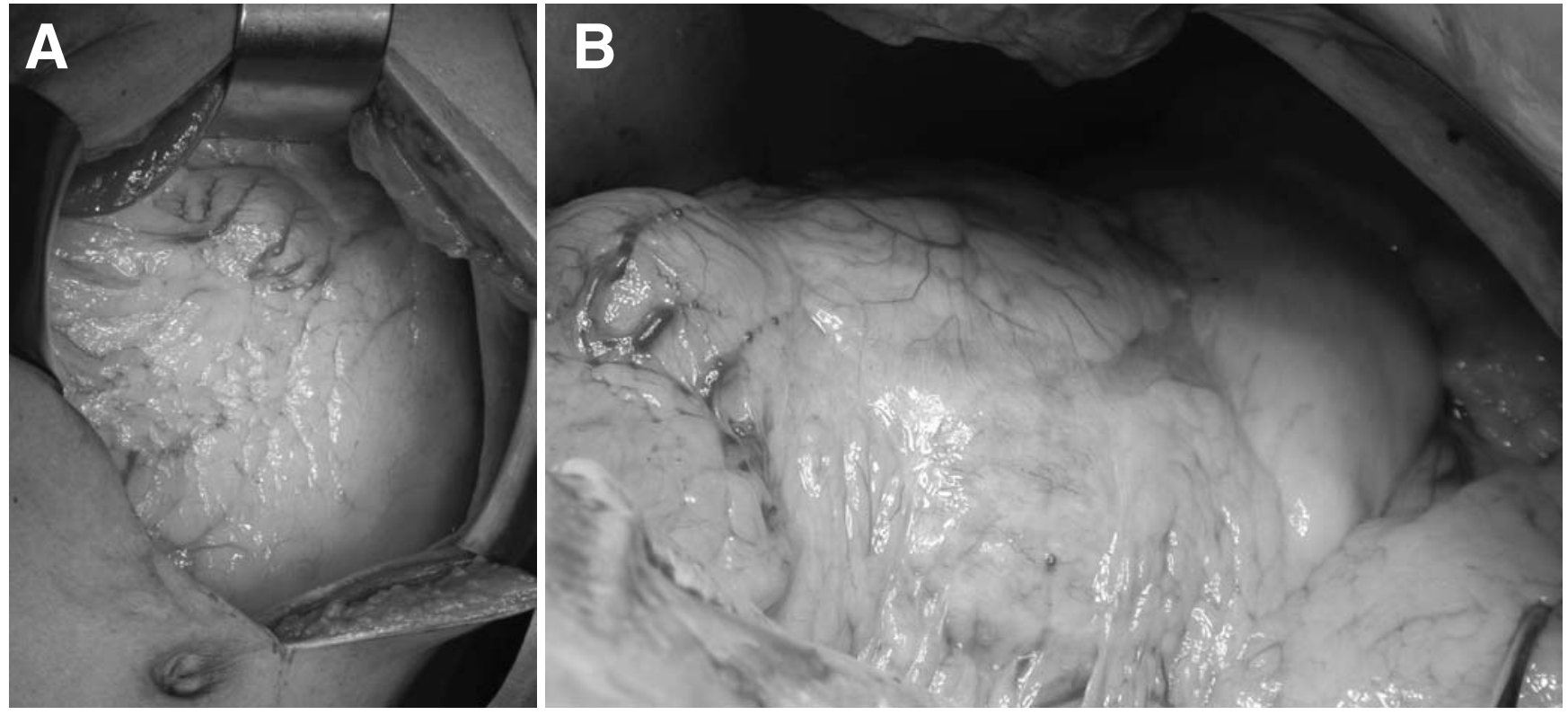

Fig. 2A,B. Intraoperative findings: peritoneal dissemination was not detected. Cytology after peritoneal lavage was class III

and its macroscopic type was 3. Biopsy of the specimen revealed the tumor was a poorly differentiated adenocarcinoma. Staging laparoscopy was performed on October 25, 2002, and revealed massive peritoneal dissemination. Cytology of the ascites was class V.

\section{Neoadjuvant chemotherapy}

The body surface area of the patient was $1.64 \mathrm{~m}^{2}$. Two courses of neoadjuvant chemotherapy with S-1 were administered, at $120 \mathrm{mg} /$ day for 28 days as one course, with a 14-day interval.
As for adverse effects, the patient suffered from grade 3 diarrhea at the end of the second course of the chemotherapy. Abdominal CT scan revealed a remarkable decrease in ascites and diminished peritoneal dissemination after the two courses of neoadjuvant chemotherapy (Fig. 1B). The sizes of the lymph nodes of the lesser curvature had not changed.

\section{Intraoperative findings}

Laparotomy was performed on January 24, 2003 (Fig. 2). The peritoneal dissemination had disappeared 


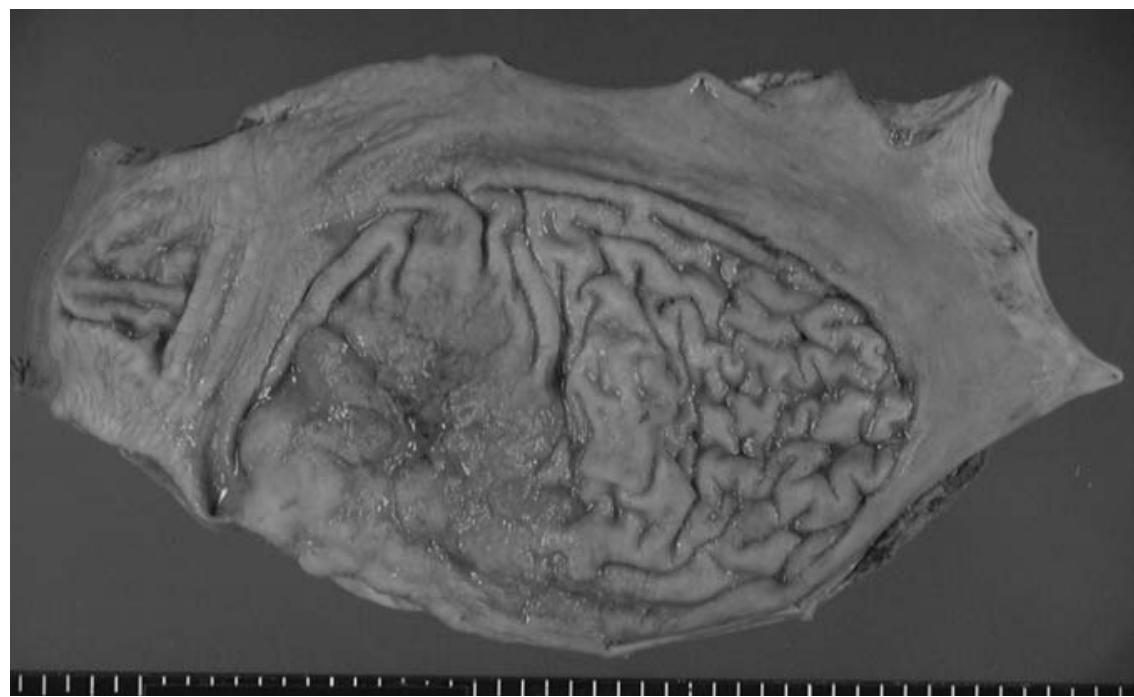

Fig. 3. Resected specimen: a type 3 tumor was seen in the greater curvature in the middle- to lower-third portion of the stomach
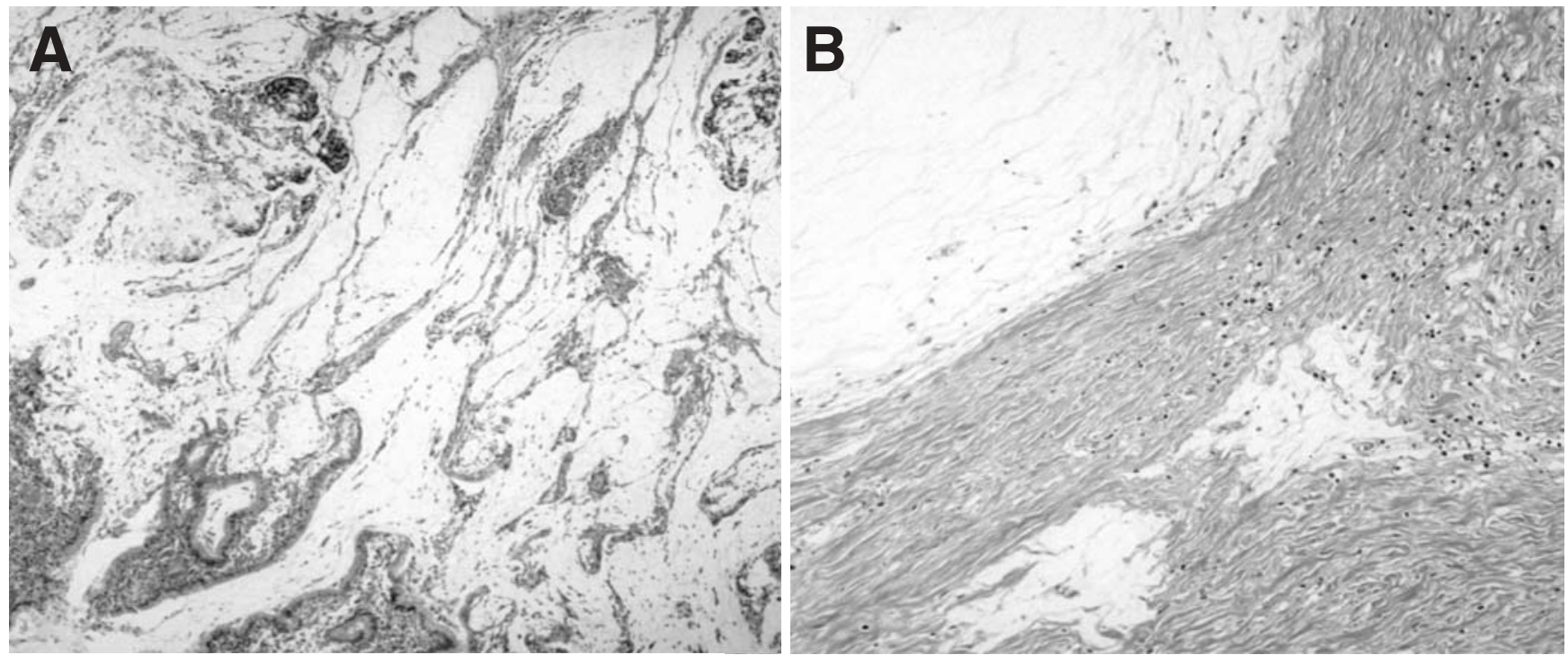

Fig. 4A,B. Microscopic findings of the resected stomach. A Pathological diagnosis is mucinous adenocarcinoma of the stomach. B Fibrotic change of the tumor tissue, caused by the chemotherapy, is observed. A H\&E, $5 \times 10 ; \mathbf{B} 5 \times 10$

macroscopically, and cytology of the peritoneal lavage fluid was class III. There was no evidence of lymph node swelling to indicate metastases. Total gastrectomy, with D2 lymph node dissection, was performed. Reconstruction of the alimentary tract was accomplished with a Roux-en-Y anastomosis with an antecolic loop.

\section{Histological diagnosis}

The histological diagnosis and the responses to the neoadjuvant chemotherapy were evaluated based on the general rules of gastric cancer study in surgery and pathology in Japan [5]. The resected specimen is shown in Fig. 3. The pathological diagnosis of the tumor was mucinous adenocarcinoma of the stomach (Fig. 4A). The number of dissected lymph nodes confirmed by histological examination was 47. Microscopic lymph node metastases were seen at the following lymph nodes: one metastasis was at the left paracardial (no. 2), two were at the lesser curvature (no. 3), and four were at the right gastroepiploic (no. 4d) lymph node group. His final diagnosis was as follows: gastric carcinoma, MLU, type 3, T2(SS), P0, H0, M0, N3, cyo, stage IV. The pathological response to chemotherapy was grade 1 (Fig. 4B). 


\section{Postoperative course}

The patient's postoperative course was uneventful. He was discharged on the twentieth postoperative day. Oral intake of uracil plus futrafur (UFT; Taiho Pharmaceutical, Tokyo, Japan) as adjuvant chemotherapy was started after he was discharged, but it was discontinued because of diarrhea. His clinical course was observed with follow-up at an outpatient clinic, without chemotherapy. He has survived for more than 23 months without any evidence of recurrence. Oral intake of S-1 ( $80 \mathrm{mg} /$ day) was restarted, but diarrhea precluded this adjuvant therapy.

\section{Discussion}

The prognosis of pathological stage IV gastric cancer with peritoneal dissemination has been poor. However, a change has occurred, with several clinical studies that have shown S-1 to have a high response rate, of approximately $40 \%-50 \%$ [6,7]. In combination with CDDP, $\mathrm{S}-1$ was reported to have a response rate as high as $70 \%$ for advanced gastric cancer [8]. Chemotherapy with S-1 has been considered as the first-line regimen for stage IV gastric cancer. Furthermore, neoadjuvant chemotherapy with S-1 for some patients with stage IV gastric cancer has been reported with good results $[9,10]$. Peritoneal dissemination of gastric cancer does not always translate into an inoperable condition. Neoadjuvant chemotherapy with S-1 has been reported to have had remarkable effects for some patients with peritoneal dissemination that could not otherwise have been operated curatively [8]. Yoshimizu et al. [11] reported a patient with pathological complete remission that was induced with preoperative chemoradiotherapy with S-1 and low-dose CDDP. Some cases of curative resection after neoadjuvant chemotherapy with $\mathrm{S}-1$ have also been reported [12-14]. It is unclear whether a better prognosis exists if neoadjuvant chemotherapy removes the peritoneal dissemination macroscopically before curative surgical resection is performed. Also, it is unclear whether neoadjuvant chemotherapy is superior to adjuvant chemotherapy after surgery $[8,15]$.

In our patient, the tumor had massive peritoneal dissemination, and its histological type was mainly mucinous gastric carcinoma. In general, this histological type has been reported to be more advanced in stage at the time of diagnosis, with a higher rate of peritoneal dissemination than nonmucinous gastric carcinomas [1618]. Some reports indicate no difference in prognosis between patients with mucinous and nonmucinous gastric cancer $[19,20]$. However, in the recent literature, the prognosis of mucinous gastric carcinomas has been reported to be worse than that of nonmucinous carcinomas, because of the advanced stage at diagnosis. The 5-year survival rate was reported to be $64.7 \%$ in patients with mucinous adenocarcinoma and $75.6 \%$ in those with nonmucinous adenocarcinoma [16,17]. Furthermore, Hirota et al. [21] reported that mucinous gastric carcinoma had the worst prognosis when compared with any other histological type of gastric carcinoma. Irrespective of these negative conditions, our patient has survived for more than 23 months after surgery. Furthermore, the patient has no detectable recurrence site, and has not been receiving any anticancer agent. These findings suggest that neoadjuvant chemotherapy with S-1 suppressed the disseminated tumor cells microscopically.

For locally advanced gastric carcinomas, Allum et al. [22] reported a British trial (the MRC [the Medical Research Council] Adjuvant Gastric Infusional Chemotherapy [MAGIC] trial, EORTC) that revealed a superior prognosis for the neoadjuvant and adjuvant groups compared to the group with surgery alone. Lowy et al. [23] reported that responders to neoadjuvant chemotherapy survived longer than nonresponders. These results suggest the possibility of a microscopic downstaging effect of neoadjuvant chemotherapy. As remarkable effects of S-1 for peritoneal dissemination of gastric cancer have been reported both experimentally [4] and clinically, true results with a randomized controlled trial (RCT) are needed to understand the full extent of the survival benefit for of S-1 gastric cancer with peritoneal dissemination [24].

With the exception of cases such as the one presented here, CT cannot usually diagnose minute peritoneal dissemination precisely, even in patients with locally advanced disease. Laparoscopic diagnosis is necessary to identify candidates for neoadjuvant chemotherapy whose imaging diagnosis reveals the absence of metastases. Blackshaw et al. [25] reported that, in 21 of 100 patients with locally advanced gastric cancer, distant metastases were found on laparoscopy, which were not detected by CT. Correct staging makes it possible to rule out patients whose surgery would be noncurative before surgery. It also allocates patients to neoadjuvant chemotherapies that are considered highly effective for advanced gastric cancers.

It is most important to determine whether curative surgery after chemotherapeutic downstaging provides a better prognosis than curative surgery alone. We await the results of RCTs. It is true that new anticancer agents [26] for gastric cancer are available. However, the oral intake of chemotherapeutic agents is desirable for the outpatient setting so that the quality of daily living is not restricted for an extended period of time prior to surgery. In the future, better neoadjuvant strategies may be established for advanced gastric carcinoma [27], but at present, oral intake of S-1 alone can be considered as one of the neoadjuvant chemotherapies for gastric cancer with peritoneal dissemination. 


\section{References}

1. Japanese Gastric Cancer Association. Gastric cancer treatment guidelines for doctors' reference (in Japanese). Tokyo: Kanehara; 2001.

2. Lee CC, Lo SS, Wu CW, Shen KH, Li AF, Hsieh MC, et al. Peritoneal recurrence of gastric adenocarcinoma after curative resection. Hepatogastroenterology 2003;50:1720-2.

3. Yoshikawa T, Kanari M, Tsuburaya A, Kobayashi O, Sairenji M, Motohashi $\mathrm{H}$, et al. Should gastric cancer with peritoneal metastasis be treated surgically? Hepatogastroenterology 2003;50:17125 .

4. Mori T, Fujiwara Y, Takiguchi S, Yasuda T, Tamura S, Yano M, et al. Evaluation of the efficacy of TS-1 for peritoneal dissemination of gastric cancer using a newly developed animal model. J Jpn Surg Soc 2002;5:448.

5. Japanese Gastric Cancer Association. Japanese classification of gastric carcinoma(in Japanese) Tokyo: Kanehara; 1999.

6. Sakata Y, Ohtsu A, Horikoshi N, Sugimachi K, Mitachi Y, Taguchi T. Late phase II study of novel oral fluoropyrimidine anticancer drug S-1 (1 M tegafur- $0.4 \mathrm{M}$ gimestat- $1 \mathrm{M}$ otastat potassium) in advanced gastric cancer patients. Eur J Cancer 1998;34: 1715-20.

7. Koizumi W, Kurihara M, Nakano S, Hasegawa K. Phase II study of S-1, a novel oral derivative of 5-fluorouracil, in advanced gastric cancer. For the S-1 Cooperative Gastric Cancer Study Group. Oncology 2000;58;191-7.

8. Yabusaki H, Nashimoto A, Tanaka O. Evaluation of TS-1 combined with cisplatin for neoadjuvant chemotherapy in patients with advanced gastric cancer (in Japanese). Gan To Kagaku Ryoho (Jpn J Cancer Chemother) 2003;30:193340.

9. Shima Y, Horimi T, Nishioka Y, Okabayashi T, Hamada M, Ishikawa $\mathrm{T}$, et al. Two resected cases of advanced gastric cancer with peritoneal dissemination after successful treatment with TS1 plus low-dose CDDP (in Japanese). Gan To Kagaku Ryoho (Jpn J Cancer Chemother) 2003;30:2115-8.

10. Kobayashi O, Konishi K, Kanari M, Cho H, Yoshikawa T, Tsuburaya A, et al. Unusual survival for more than 2 years with peritoneal metastases of gastric cancer. Gastric Cancer 2002;5:4750 .

11. Yoshimizu M, Saikawa Y, Kubota T, Akiba, Yoshida M, Otani Y, et al. Complete response of a highly advanced gastric carcinoma to preoperative chemoradiotherapy with S-1 and low-dose cisplatin. Gastric Cancer 2003;6;185-90.

12. Yoshikawa T, Kanari M, Tsuburaya A, Kobayashi O, Sairenji M, Motohashi $\mathrm{H}$, et al. Advanced gastric carcinoma successfully treated with TS-1 as neoadjuvant chemotherapy. Gastric Cancer 2000;27:171-5.
13. Iwahashi M, Nakamori M, Tani M, Yamaue H, Sakaguchi S, Nakamura M, et al. Complete response of highly advanced gastric cancer with peritoneal dissemination after new combined chemotherapy of S-1 and low-dose cisplatin: report of a case. Oncology 2001;61:16-22.

14. Mohri N, Akamo Y, Hayakawa T, Takeyama H, Manabe T. Case of advanced gastric cancer that was resectable after asctic fluid had disappeared following administration of TS-1 (in Japanese). Gan To Kagaku Ryoho (Jpn J Cancer Chemother) 2001;28:9991002.

15. Maehara Y, Baba H, Sugimachi K. Adjuvant chemotherapy for gastric cancer: a comprehensive review. Gastric Cancer 2001;4: $175-84$.

16. Kawamura H, Kondo Y, Osawa S, Nisida Y, Okada K, Isizu H, et al. A clinicopathologic study of mucinous adenocarcinoma of stomach. Gastric Cancer 2001;4:83-6.

17. Shibuya H, Nishida R, Koide S, Kurokawa J, Okita K. Clinicopathological study of mucinous gastric carcinoma. Ann Chir Gynaecol 2000;89:273-6.

18. Woo LS, Kim DY, Kim YJ, Kim SK. Clinicopathologic features of mucinous gastric carcinoma. Dig Surg 2002;19:286-90.

19. Yasuda K, Adachi Y, Shiraishi N, Yamaguchi K, Shiromizu A, Kitano S. Pathology and prognosis of mucinous gastric carcinoma. J Surg Oncol 2001;76:272-7.

20. Adachi Y, Yasuda K, Kitano S. Mucinous gastric carcinoma: is it more malignant? Gastric Cancer 2001;4:223-4.

21. Hirota T, Ochiai A, Masayuki I, Maruyama K. Significance of histological type of gastric carcinoma as a prognostic factor (in Japanese). I to Cho (Stomach and Intestine) 1991;26:1149-58.

22. Allum W, Cunningham D, Weeden S. Perioperative chemotherapy in operable gastric and lower oesophageal cancer: a randomised, controlled trial (the MAGIC trial, ISRCTN 93793971). Proc ASCO 2003;22:249.

23. Lowy AM, Mansfield PF, Leach SD, Pazdur R, Dumas P, Ajani JA. Response to neoadjuvant chemotherapy best predicts survival after curative resection of gastric cancer. Ann Surg 1999; 229:303-8.

24. Kinoshita T, Konishi M, Nakagohri T, Inoue K, Oda T, Takahashi $\mathrm{S}$, et al. Neoadjuvant chemotherapy with S-1 for scirrhous gastric cancer: a pilot study. Gastric Cancer 2003;6(Suppl 1):40-4.

25. Blackshaw GR, Barry JD, Edwards P, Allison MC, Thomas GV, Lewis WG. Laparoscopy significantly improves the perceived preoperative stage of gastric cancer. Gastric Cancer 2003;6:225-9.

26. Sakurai Y, Yoshida I, Tonomura S, Sakai W, Nakamura Y, Imazu $\mathrm{H}$, et al. Weekly administration of paclitaxel attenuated rectal stenosis caused by multiple peritoneal recurrence 8 years after the resection of gastric carcinoma. Gastric Cancer 2003;6:243-9.

27. Sakamoto J. Neoadjuvant chemotherapy: a standard treatment for locally advanced gastric cancer in the near future? Gastric Cancer 2003;6:131-3. 